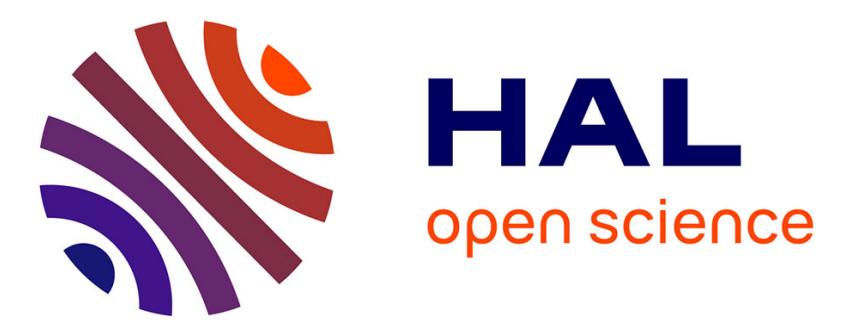

\title{
Données nouvelles sur l'âge des vestiges de chien à Pont d'Ambon, Bourdeilles (Dordogne)/New data on the age of Canis remains at Pont d'Ambon, Bourdeilles (Dordogne, France)
}

Guy Célérier, Nadine Tisnerat, Hélène Valladas

\section{To cite this version:}

Guy Célérier, Nadine Tisnerat, Hélène Valladas. Données nouvelles sur l'âge des vestiges de chien à Pont d'Ambon, Bourdeilles (Dordogne)/New data on the age of Canis remains at Pont d'Ambon, Bourdeilles (Dordogne, France). PALEO : Revue d'Archéologie Préhistorique, 1999, 11 (1), pp.163165. 10.3406/pal.1999.1253 . hal-02506269

\section{HAL Id: hal-02506269 \\ https://hal.science/hal-02506269}

Submitted on 8 Feb 2021

HAL is a multi-disciplinary open access archive for the deposit and dissemination of scientific research documents, whether they are published or not. The documents may come from teaching and research institutions in France or abroad, or from public or private research centers.
L'archive ouverte pluridisciplinaire HAL, est destinée au dépôt et à la diffusion de documents scientifiques de niveau recherche, publiés ou non, émanant des établissements d'enseignement et de recherche français ou étrangers, des laboratoires publics ou privés. 


\section{Données nouvelles sur l'âge des vestiges de chien à Pont d'Ambon,} Bourdeilles (Dordogne)/New data on the age of Canis remains at Pont d'Ambon, Bourdeilles (Dordogne, France)

\section{Guy Célérier, Nadine Tisnerat, Hélène Valladas}

\section{Abstract}

New data on the age of Canis remains at Pont d'Ambon, Bourdeilles (Dordogne, France). After a short historical record of the discovery, this note presents the results of analysis made on Canis remains from the Pont d'Ambon site which confirm the palaeolithic age dating in a Laborian context.

\section{Citer ce document / Cite this document :}

Célérier Guy, Tisnerat Nadine, Valladas Hélène. Données nouvelles sur l'âge des vestiges de chien à Pont d'Ambon, Bourdeilles (Dordogne)/New data on the age of Canis remains at Pont d'Ambon, Bourdeilles (Dordogne, France). In: Paléo. N. 11,1999. pp. 163-165;

doi : https://doi.org/10.3406/pal.1999.1253

https://www.persee.fr/doc/pal_1145-3370_1999_num_11_1_1253

Fichier pdf généré le 29/03/2019 


\title{
DONNÉES NOUVELLES SUR L'ÂGE DES VESTIGES DE CHIEN A PONT D'AMBON, BOURDEILLES, (Dordogne)
}

\author{
Guy CÉLÉRIER(1) Nadine TISNÉRAT ${ }^{(2)}$ \& Hélène VALLADAS ${ }^{(2)}$
}

\begin{abstract}
Résumé : Cette note est destinée à faire connaître une mesure d'âge réalisée sur les vestiges de Chien mis au jour sur le site de Pont d'Ambon.

Après un bref historique de la découverte, les résultats de l'analyse sont exposés. Ils cónfirment l'authenticité paléolithique de ces restes et apportent en même temps une date en accord avec le contexte matériel laborien.

Mots-clés : Pont d'Ambon, Chien, datation, Laborien, Tardiglaciaire.

Abstract: New data on the age of Canis remains at Pont d'Ambon, Bourdeilles (Dordogne, France). After a short historical record of the discovery, this note presents the results of analysis made on Canis remains from the Pont d'Ambon site which confirm the palaeolithic age dating in a Laborian context.
\end{abstract}

Keywords : Pont d'Ambon, Canis. Dating, Laborian, Tardiglacial

\section{Historique}

La campagne de fouilles de juillet 1976 menée sur le site de Pont d'Ambon entraînait la découverte de vestiges de Canidé dans un niveau inférieur de la couche 2 , carré $\mathrm{K}$ 7. La détermination spécifique de ceux-ci permettait à $F$. Delpech, paléontologue, d'assurer que l'ensemble des pièces appartenait à un Chien adulte de taille modeste.

Le dégagement des restes, rendu difficile par l'extrême fragilité des lames scapulaires et la forte cohésion du sédiment argileux brun très foncé dans lequel ils étaient inclus, a permis de bien les distinguer du contenu grumeleux, jaunâtre, d'un terrier fossile effondré qui leur était sus-jacent. La situation stratigraphique se révélait parfaitement claire au moment de la fouille ; néanmoins, ce voisinage allait constituer un point d'achoppement et faire douter de l'âge paléolithique des vestiges. Pourtant, de nombreuses évidences paraissaient constituer des critères incontestable : accumulation dans une zone restreinte d' une grande variété d'éléments osseux post crâniens, fracturations diaphysaires, présence dans la fosse sous-épineuse de la scapula gauche de profondes stries parallèles correspondant probablement à des traces de décarnisation.

L'ensemble était composé des deux scapulas, un fragment distal d'humérus droit (non représenté ici), les extrémités proximales fracturées des cubitus droit et gauche, une portion proximale de radius gauche ainsi que la plus grande partie d'une patte postérieure (fig.).

L'intérêt exceptionnel de cette découverte incitait le responsable du chantier (G.C.) et F. Delpech à la faire connaître à la communauté archéologique (Célérier et Delpech, 1978).

Les conclusions de cet article restaient ouvertes sur de futures découvertes dans l'incapacité d'expliquer les raisons de la petite taille de cet individu sans qu'intervienne la notion de domestication.

En 1986, au cours du $5^{\text {me }}$ Congrès International d'Archéozoologie de Bordeaux, J.D. Vigne, dans une

(1) 67, rue Combe-des-Dames, 24000 Périgueux.

(2) Laboratoire des Sciences du Climat et de l'Environnement, Avenue de la Terrasse, 91198 Gif-sur-Yvette. 
intervention consacrée aux Canidés d'Europe et à leur domestication, rejetait de façon péremptoire et définitive les restes du Canidé de Pont d'Ambon au prétexte qu'ils n'appartenaient pas à la couche azilienne (J.D. Vigne et M.C. Marinval-Vigne, 1988). Les preuves de l'authenticité ne pouvaient, dans ce cas, être apportées que par une datation directe des vestiges.

\section{Mesure d'âge}

Une analyse carbone 14 en spectrométrie de masse par accélérateur a alors été effectuée sur le Tandétron de Gif-sur-Yvette. Le fragment diaphysaire provenant de la zone fracturée du cubitus (environ $2 \mathrm{~g}$ ) a subi un traitement chimique (Nelson, 1990) destiné à éliminer les contaminations et transformer le carbone des acides aminés du collagène en gaz carbonique. Ce gaz, équivalent à $1,9 \mathrm{mg}$ de carbone, a été réduit catalytiquement en graphite selon le protocole décrit par Arnold et alii (1987). Après avoir été pastillé, l'échantillon de graphite a été analysé par le Tandétron.

L'âge obtenu est :

GifA $99102: 10730 \pm 100$ ans B.P.

A l'évidence, cette datation confirme l'authenticité paléolithique des restes de Canidé de Pont d'Ambon; elle s'accorde avec d'autres témoignages provenant de l'Europe occidentale et singulièrement avec celui des grottes Jean-Pierre à Saint-Thibaud-de-Couz (Savoie) (Chaix, 1994 ; (J.D. Vigne et M.C. Marinval-Vigne op. cit.; Baales et Street, 1998).

Dans le même temps, elle apporte des éléments très importants sur la situation chronologique de l'industrie à forte connotation "laborienne" de la couche 2, puisque cette date est proche de celle du Laborien du Lot-etGaronne ( Ly 1401 : $10350 \pm 340$ B.P.). En effet, l'écart qui existait entre la première datation de la couche 2 de Pont d'Ambon (Gif $3740: 9640 \pm 120$ B.P.) et celle de La Borie del Rey restait difficilement explicable compte tenu de la forte similitude des cultures matérielles (Célérier, 1998). Et ce, d'autant que les découvertes récentes, dans la région parisienne d'un niveau culturel semblable au Closeau (Rueil-Malmaison) livraient, pour le locus 25, les dates de 10755 et 10885 B.P. (P. Bodu, D.F.S. de sauvetage d'urgence, 1998).

La date obtenue sur les vestiges de Chien, importante pour l'argumentation des phénomènes conjecturaux du proto-élevage de ces animaux revêt en outre un singulier intérêt, car elle permet de confirmer l'unité chronologique de ces industries "laboriennes" en même temps qu'on observe au fil des découvertes actuelles, leur relative dispersion spatiale.

\section{BIBLIOGRAPHIE}

ARNOLD M., BARD E., MAURICE P., DUPLESSY J.C., $1987:{ }^{14} \mathrm{C}$ dating with the Gif/Yvette Tandetron accelerator: status report. Nuclear Instruments and Methods in Physics Research; B29, p. 120-123.

BAALES M. \& STREET M., 1998 : Late Palaeolithic Backed-point assemblages in the northern Rhineland: current research and changing views. Notae Praehistoricae, t. 18, p. 77-92.

BARD E., ARNOLD M., FAIRBANKS R.G., HAMELIN B., 1993 : Radiocarbon, t. 35, p. 191-199.

CÉLÉRIER G., 1998 : L'abri sous roche de Pont d'Ambon à Bourdeilles ( Dordogne, France). Perspective synthétique. Paléo, $n^{\circ} 10$, p. 233-264, ill.

CÉLÉRIER G. et DELPECH F., 1978 : Un chien dans l'Azilien de «Pont d'Ambon" (Dordogne) ? Bull. de la Soc. Préh. Franç. T. 75, p. 212-215, ill.

CHAIX L., 1994 : in BINTZ P. et alii. Les grottes JeanPierre 1 et 2 à Saint-Thibaud-de-Couz (Savoie). Gallia Préhistoire, t. 36, p. 145-266, ill.

NELSON D.E., 1990 : A new method for carbon isotopic analysis of protein. Science, 251, p. 552-554.

VIGNE J.D. et MARINVAL-VIGNE M.C., 1988 : Quelques réflexions préliminaires sur les Canidés mésolithiques de Noyen-sur-Seine (France) et sur la domestication du Chien en Europe occidentale. In Actes du 5ème Congrès International d'Archéozoologie ; Bordeaux, Août 1986. Archaeozoologia, vol. II/1.2, p. 153-164.

Remerciements : Nous adressons de vifs remerciements à J.J. Cleyet-Merle, conservateur en chef du Musée National de Préhistoire, Les Eyzies, de l'intérêt qu'il a montré pour tout ce qui concerne Pont d'Ambon, à S. Madelaine pour son aide précieuse lors du prélèvement. 

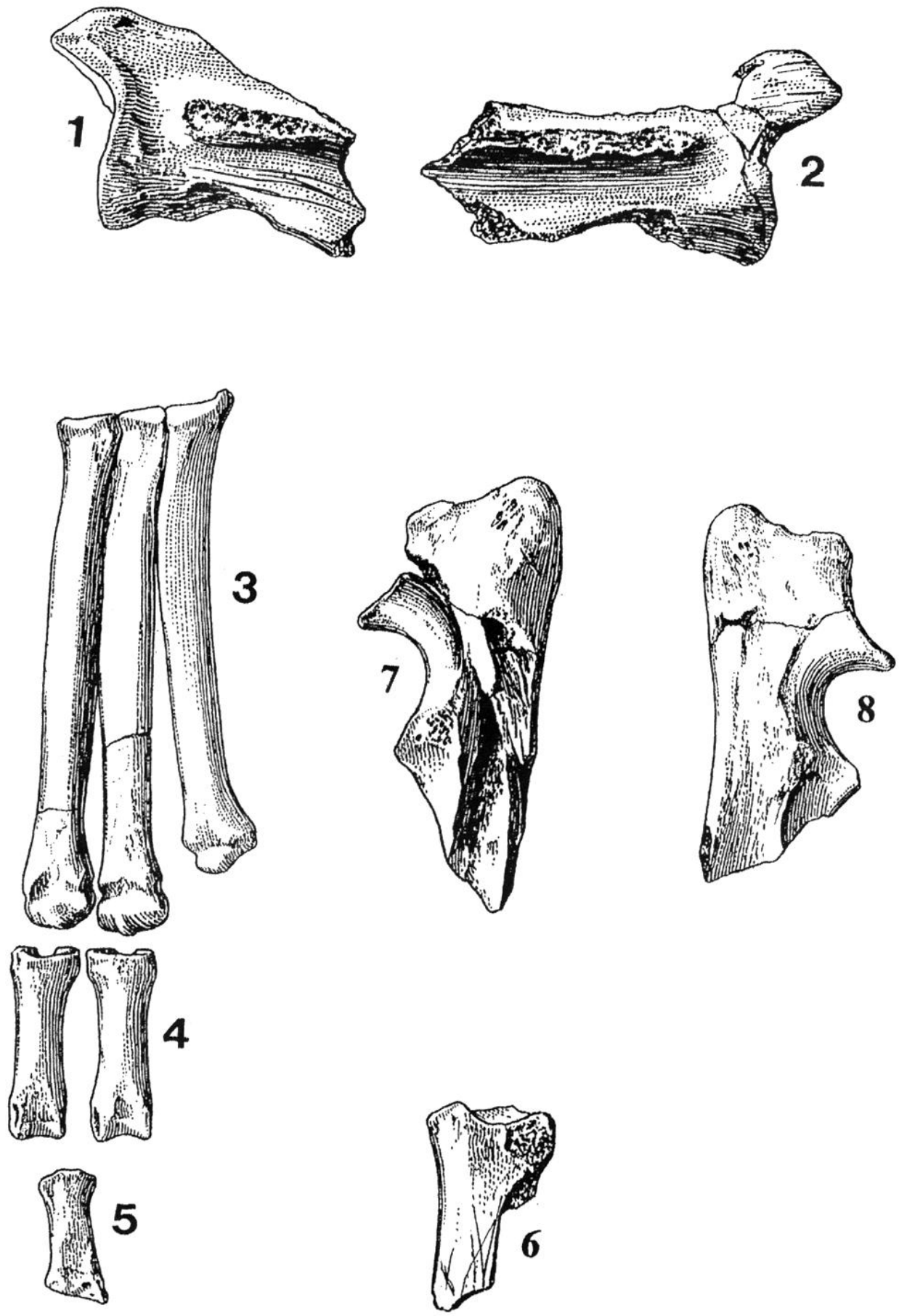

PONT D'AMBON (Bourdeilles, Dordogne) 1976, K 7351 - Vestiges de Canis : 1 et 2 : Fragments inférieurs des scapulas droite et gauche, vue latérale. - $[3,4,5]$ : éléments d'une même patte postérieure gauche; 3 , troisième, quatrième et cinquième métatarsiens ; 4 , deux premières phalanges ; 5 , deuxième phalange. - 6 , fragment proximal de radius gauche, vue dorsale. - 7, fragment proximal de cubitus gauche. - 8, extrémité proximale de cubitus droit, vue latérale.

Grandeur nature (dessins de Pierre Laurent).

PONT D'AMBON (Bourdeilles, Dordogne) 1976, K 7351 - Canis remains : 1 and 2 : inferior fragments of scapula right and left, lateral view. - $[3,4,5]$ : elements of one left posterior paw; 3, third, fourth and fifth metatarsal; 4, two first phalanxes; 5, second phalanx. - 6, proximal fragment of left radius, dorsal view. - 7 proximal fragment of left ulna. - 8, proximal extremity of straight ulna, lateral view.

Full scale (drawings by Pierre Laurent). 